\title{
Accurate Identification of Reentrant Circuit and Critical Isthmus of an Atrial Tachycardia Over the Posterior Wall of the Left Atrium Requiring a 1.4-second Single Radiofrequency Energy Application
}

\author{
HAKOP HRACHIAN, MD ${ }^{1}$ and JULIANA RIOS, BS, CEPS, CCDS $^{2}$
}

${ }^{1}$ Mount Sinai Medical Center, Miami Beach, FL, USA

${ }^{2}$ Abbott, Chicago, IL, USA

KEYWORDS. Atrial tachycardia, high-density mapping, radiofrequency ablation, repeat ablation.
ISSN 2156-3977 (print) ISSN 2156-3993 (online) CC BY 4.0 license

(C) 2021 Innovations in Cardiac Rhythm Management
A 61-year-old man with longstanding persistent atrial fibrillation (AF) since 2010 presented to the clinic, having undergone cardioversion for AF in 2016 and AF ablation (pulmonary vein isolation, posterior left atrium isolation, and cavotricuspid isthmus ablation) in July 2017. He developed atrial tachycardia (AT) a few months after the AF ablation procedure and underwent AT ablation (LA posterior wall) in October 2017. However, he experienced early recurrence, requiring a second cardioversion (for AT) and treatment with dofetilide in 2018. He remained primarily in sinus rhythm until September 2020, when he developed persistent AT again, which recurred after a third cardioversion attempt in early October 2020.

Dr. Hrachian reports the reception of research grants from Abbott and is also consultant for Abbott. Ms. Juliana Rios is an employee of Abbott. Both authors report no equity relationship with Abbott. Address correspondence to: Hakop Hrachian, MD.

Email: hakop11@msn.com.
In late October 2020, the patient underwent repeat AT ablation using the Advisor ${ }^{\mathrm{TM}}$ HD Grid Mapping Catheter, Sensor Enabled ${ }^{\mathrm{TM}}$, which confirmed macro-reentrant AT originating from the posterior wall of the left atrium (Video 1). A very narrow isthmus was identified precisely; delivery of one radiofrequency energy application with $35 \mathrm{~W}$ of power terminated the tachycardia in 1.4 seconds.

However, postablation mapping showed scattered areas of live tissue over the posterior wall communicating with the left atrium. Mapping with the Advisor ${ }^{\mathrm{TM}} \mathrm{HD}$ Grid catheter was able to identify the reentrant circuit accurately and further ablation was performed in sinus rhythm to achieve complete isolation of the posterior wall. In addition, all remaining potentials were eliminated, having been missed using other catheters during the previous two ablation sessions. Isolation persisted after 30 minutes of observation and the infusion of isoproterenol during the postablation period. 


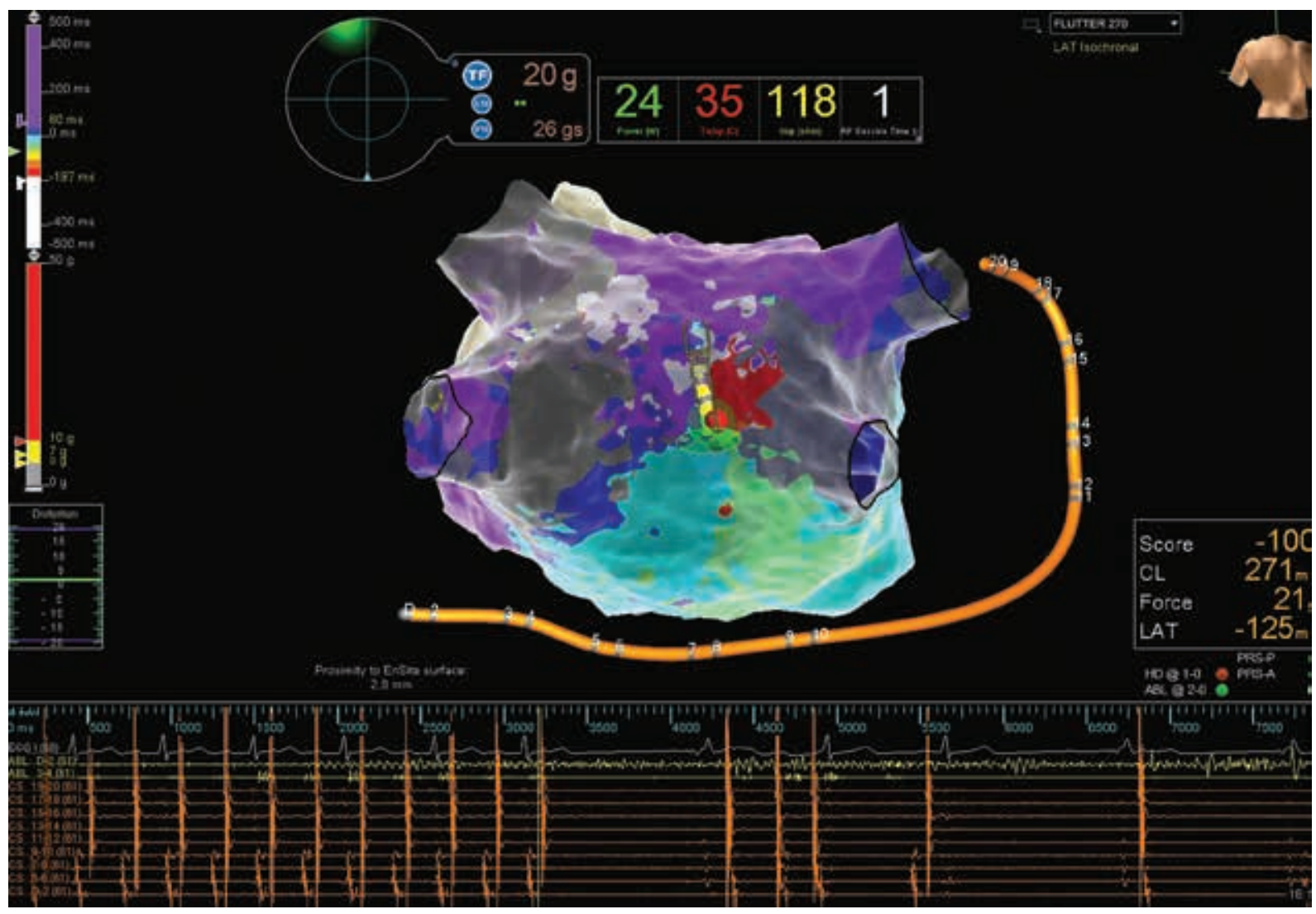

Figure 1: Left atrial posterior wall. 